\title{
Nonparametric Confidence Interval for Quantiles
}

\author{
Mohammad Bolbolian Ghalibaf \\ Department of Statistics, Faculty of Mathematics and Computer Sciences \\ Hakim Sabzevari University, Sabzevar, Iran \\ m.bolbolian@hsu.ac.ir
}

\begin{abstract}
Quantiles, which are also known as values-at-risk in finance, frequently arise in practice as measures of risk. Confidence intervals for quantiles are typically constructed via large sample theory or the sectioning.

One of the ways for achieving the confidence interval for quantiles is direct use of a central limit theorem. In this approach, we require a good estimator of the quantile density function. In this paper, we consider the nonparametric estimator of the quantile density function from Soni et al. (2012) and we obtain confidence interval for quantiles. In the following, by using simulation, the coverage probability and mean square error of this confidence interval is calculated. Also, we compare our proposed approach with alternative approaches such as sectioning and jackknife.
\end{abstract}

Keywords: Bandwidth, Jackknife Estimator, Kernel Function, Nonparametric Confidence Interval, Quantile Density Function, Quantile Function, Sectioning.

\section{Introduction}

The quantile function $Q \equiv F^{-1}$ associated with a distribution function $F$ defined as

$$
Q(u)=F^{-1}(u)=\inf \{x ; F(x) \geq u\}, \text { for } 0<u<1,
$$

is sometimes the object of more direct interest than the $F$ itself. The use of quantiles spans a wide range of fields, especially in finance, which are known as values-at-risk, nuclear engineering and project planning (Nakayama, 2012).

Assuming $F$ has a positive derivative $F^{\prime}(x)=f(x)$ on its domain, define

$$
q(u)=Q^{\prime}(u)=\frac{1}{f(Q(u))},
$$

to be the quantile density function by Parzen (1979), and earlier dubbed the sparsity index by Tukey (1965). The quantile density function is of much practical relevance mainly because it appears as part of the asymptotic variance of empirical quantiles.

The basic concept for deriving the confidence Interval (CI) for quantiles is well known. The first time Woodruff (1952) presented a method for estimating a CI for quantiles. The approach consists of three steps: a point estimate of the cumulative distribution function, a CI for the point estimate, and converting it into $\mathrm{CI}$ for the quantile.

Nowadays, there are several approaches for deriving CI for quantiles, but no consensus about the best practice. In the following, we will express three attempts on the problem. The first attempt is direct use of a central limit theorem (CLT). We try to improve it by using nonparametric estimator of the quantile density function. The second attempt is to use sectioning or batching to calculate both a point estimate of the quantile and an 
estimate of the variance of the point estimate. The last attempt is to apply a jackknife approach to reducing the bias of previously method (based on sectioning).

In this paper, we consider the first approach and by using nonparametric estimator of the quantile density function introduced by Soni et al. (2012) construct a CI for quantile. Then we compare results of three methods numerically.

The layout of the paper is as follows: In Section 2, we express three methods to achieve CI for quantile. In Section 3, we represent smooth estimator of the quantile density function from Soni et al. (2012) and using it construct a CI for quantile. Section 4 contains the results of the simulation. In this section, we compare coverage probability (CP), mean square error (MSE) and the expected length of CI in our introduced approach with those in two other approaches. The conclusion is given in Section 5 and all tables appear in the appendix.

\section{Confidence Interval Attempts}

In the following we will review three attempts to achieve CI for quantiles.

\subsection{Direct use of a CLT}

A common approach to developing a $\mathrm{CI}$ is to first show that the quantile estimator satisfies a CLT, and then replace the variance constant in the CLT with a consistent estimator of it to construct a CI. We will use this approach to introduce nonparametric CI for quantile. For this purpose, we use the CLT for quantile estimator from Serfling (1980).

Suppose $X_{1}, \ldots, X_{n}$ be $n$ identically independent distribution (i.i.d.) random variables from a distribution $F$ and $X_{(1)} \leq \ldots \leq X_{(n)}$ be the order statistics corresponding to $X_{1}, \ldots, X_{n}$. Let $F_{n}(x)$ be the usual empirical distribution function, i.e.,

$$
F_{n}(x)=\frac{1}{n} \sum_{i=1}^{n} I\left(X_{i} \leq x\right) \text {, }
$$

the sample estimator corresponding to $Q(u)$ is defined by

$$
\hat{Q}_{n}(u)=F_{n}^{-1}(u)=\sum_{i=1}^{n} X_{(i)} I_{\left(\frac{i-1}{n}, \frac{i}{n}\right]}(u), \text { for } 0<u<1 .
$$

Serfling (1980) shows that $\hat{Q}_{n}(u)$ is asymptotically normal with mean $Q(u)$ and variance $\frac{\sigma^{2}}{n}$, where

$$
\sigma=\frac{\sqrt{u(1-u)}}{f(Q(u))}=\sqrt{u(1-u)} q(u) .
$$

This leads to a large sample $100(1-\alpha) \%$ CI for quantile $Q(u)$ of the form

$$
\hat{Q}_{n}(u) \pm z_{1-\frac{\alpha}{2}} \frac{\sigma}{\sqrt{n}}
$$


Unfortunately, this CI is not implementable in practice since $q(u)$ is typically unknown, and then so is $\sigma$. If we have a consistent estimator for $q(u)$ (i.e., $q_{n}(u) \rightarrow q(u)$ as $\left.n \rightarrow \infty\right)$, then we can replace $\sigma$ in Equation (1) by $S_{n}=\sqrt{u(1-u)} q_{n}(u)$ to obtain

$$
\hat{Q}_{n}(u) \pm z_{1-\frac{\alpha}{2}} \frac{S_{n}}{\sqrt{n}}
$$

as another approximate $100(1-\alpha) \%$ CI for quantile $Q(u)$.

Generality, in this method there are two sources of error to build the CI for $Q(u)$. The first source, estimator of the quantile function and another source, estimator of the quantile density function. To view the various estimators for the $u$-th quantile refer to Steitnberg (1983). The estimation of the quantile density function for the first time have been suggested by Siddiqui (1960) and studied by Bloch and Gastwirth (1968), Bofinger (1975), Reiss (1978), Sheather and Maritz (1983), Babu (1986), Falk (1986), Welsh (1988), Jones (1992), Soni et al. (2012) and Chesneau et al. (2016).

\subsection{Sectioning}

Sectioning or batching is a very general methodology for constructing CI for a performance measure $\alpha$. All the performance measures considered thus far in the course can be handled via the use of sectioning (for a detailed discussion of sectioning see Lewis and Orav, 1988). To illustrate the idea, suppose that we wish to construct a CI for quantile $Q(u)$. We suppose that the total number of independent replications $n$ of our simulation experiment takes the form $n=m k$, where $m$ corresponds to the number of sections. Think of $m$ as being relatively small (say 10 to 20), with the number of observations $k$ per section being very large.

Let $Q_{n(i)}(u)$ be the estimator for quantile $Q(u)$ based on all the observations $\left\{X_{j}:(i-1) k+1 \leq j \leq i k\right\}$ associated with the $i$-th section. Observe that the estimators $Q_{n(1)}(u), \ldots, Q_{n(m)}(u)$ are i.i.d. (since each estimator is based on a statistically identical, independent section of observations). By the previous CLT,

$$
Q_{n(i)}(u) \stackrel{D}{\sim} N_{i}\left(Q(u), \frac{\sigma^{2}}{k}\right) \text { for } 1 \leq i \leq m,
$$

where the normal random variables $N_{1} \ldots, N_{m}$ are independent. In other words, the section estimators $Q_{n(1)}(u), \ldots, Q_{n(m)}(u)$ behave, for large $n$ (or equivalently, large $k$ ), like i.i.d. normal random variables with mean $Q(u)$ (the quantity we wish to estimate) and unknown variance $\frac{\sigma^{2}}{k}$. Therefore if $n$ is large (so that the CLTs are good approximations), it follows that an approximate $100(1-\alpha) \%$ CI for quantile $Q(u)$ is

$$
\bar{Q}_{n} \pm t_{m-1,1-\frac{\alpha}{2}} \sqrt{\frac{V_{n}}{m}}
$$

where 


$$
\begin{aligned}
& \bar{Q}_{n}=\frac{1}{m} \sum_{i=1}^{m} Q_{n(i)} . \\
& V_{n}=\frac{1}{m-1} \sum_{i=1}^{m}\left(Q_{n(i)}-\overline{Q_{n}}\right)^{2} .
\end{aligned}
$$

and $t_{m-1,1-\frac{\alpha}{2}}$ is percentile $\left(1-\frac{\alpha}{2}\right)$ from Student-t distribution with $m-1$ degrees of freedom.

Remark 1. It is important, in using the above Student- $t$ CI, to use the divisor $m-1$ (rather than $m$ ) in computing $V_{n}$, because the number of sections $m$ will typically be relatively small.

\subsection{The Jackknife Estimator and Sectioning}

$\hat{Q}_{n}$ and $\bar{Q}_{n}$ in CLT and sectioning methods are two estimators of quantile $Q(u)$ respectively, but $\overline{Q_{n}}$ has a bias that is roughly $\$ \mathrm{~m} \$$ times as large as that of $\hat{Q}_{n}$. The large bias of $\bar{Q}_{n}$ makes it an undesirable estimator for quantile $Q(u)$ and renders this approach to constructing CI for quantile $Q(u)$ unsuitable without some further modification. We can apply a jackknife approach to reducing the bias of previously described CI methodology based on sectioning (for more details see Lewis and Orav (1988) and Nelson (1990)).

Suppose that $Q_{n}$ be the sample estimator for $Q(u)$ based on all $n$ observations and $Q_{n}^{o}(i)$ be the sample quantile associated with all the $n$ replications, except those in the $i$ th section (i.e., replications indexed from $(i-1) k+1$ through $i k$, for $i=1, \ldots, m)$. We compute the $m$ pseudo-values

$$
Q_{n}(i)=m Q_{n}-(m-1) Q_{n}^{o}(i) \text { for } 1 \leq i \leq m .
$$

Jackknife sectioning estimator for quantile $Q(u)$ defined by

$$
Q_{n}^{J}=\frac{1}{m} \sum_{i=1}^{m} Q_{n}(i)
$$

and Jackknife variance estimator computed by

$$
V_{n}^{J}=\frac{1}{m-1} \sum_{i=1}^{m}\left(Q_{n}(i)-Q_{n}^{J}\right)^{2}
$$

Therefore

$$
Q_{n}^{J} \pm t_{m-1,1-\frac{\alpha}{2}} \sqrt{\frac{V_{n}^{J}}{m}},
$$

is an approximate $100(1-\alpha) \%$ CI for quantile $Q(u)$, where $t_{m-1,1-\frac{\alpha}{2}}$ is percentile $\left(1-\frac{\alpha}{2}\right)$ from Student-t distribution with $m-1$ degrees of freedom. 


\section{Nonparametric Confidence Interval}

In this section, we represent kernel estimator of the quantile density function from Soni et al. (2012). Then we express introduction to the kernel function and bandwidth and finally by using estimator of the quantile density function, we introduce a CI for the quantile $Q(u)$.

\subsection{Estimator of the Quantile Density Function}

Soni et al. (2012) introduce a smooth estimator of the quantile density function. This estimator is made based on kernel function. Based on sample $X_{1}, \ldots, X_{n}$, they propose a smooth estimator of the quantile density function as

$$
q_{n}(u)=\frac{1}{h(n)} \int_{0}^{1} \frac{K\left(\frac{t-u}{h(n)}\right)}{f_{n}\left(\hat{Q}_{n}(t)\right)} d t,
$$

where $K($.$) is a kernel and h(n)$ is the bandwidth sequence. The estimator (2) can also be written as

$$
q_{n}(u)=\frac{1}{h(n)} \sum_{i=1}^{n} \frac{1}{f_{n}\left(X_{(i)}\right)} \int_{S_{i-1}}^{S_{i}} K\left(\frac{t-u}{h(n)}\right) d t,
$$

where $S_{i}$ is the proportion of observations less than or equal to $X_{(i)}$.

Generality, for small $S_{i}-S_{i-1}$, we use the mean value theorem to get

$$
q_{n}(u)=\frac{1}{n h(n)} \sum_{i=1}^{n} \frac{K\left(\frac{S_{i}-u}{h(n)}\right)}{f_{n}\left(X_{(i)}\right)} .
$$

Kernel estimator is characterized by the kernel function, $K($.$) , which determines the$ shape of the weighting function, and the bandwidth, $h(n)$, which determines the "width" of the weighting function and hence the amount of smoothing. The two components determine the properties of the estimator. Considerable research has been carried out (and continues to be done) on the question of how one should select $K($.$) and h(n)$ in order to optimize the properties of the estimator. This issue will be discussed in the following. The kernel $K($.$) is a real valued function satisfying the following properties:$

$$
\begin{array}{ll}
\text { i. } & K(u) \geq 0 \text { for all } u \text {; } \\
\text { ii. } & \int_{-\infty}^{\infty} K(u) d u=1 ;
\end{array}
$$

iii. $\quad K($.$) has finite support, that is \mathrm{K}(\mathrm{u})=0$ for $|u|>c$ where $c>0$ is some constant;

iv. $\quad K($.$) is symmetric about zero;$

v. $\quad K($.$) satisfies Lipschitz condition, viz there exists a positive constant M$ such that $|K(u)-K(v)| \leq M|u-v|$. 
Several types of kernel functions are commonly used. Prakasa Rao (1983) shows that Epanechnikov kernel is the optimal kernel and efficiency of kernel functions relative to Epanechnikov kernel measure. This kernel is defined as

$$
K(u)=\frac{3}{4}\left(1-u^{2}\right) I(|u| \leq 1) .
$$

In Equation (3), the parameter $h$ is called the bandwidth or smoothing constant. It determines the amount of smoothing applied in estimating kernel. Selection of the bandwidth of kernel estimator is a subject of considerable research. Four popular methods commonly used are: subjective selection, selection with reference to some given distribution, cross-validation and "plug-in" estimator.

These bandwidth selectors represent only a sample of the many suggestions that have been offered in the recent literature. Some alternatives are described in Wand and Jones (1995) in which the theory is given in more details.

Unlike the above methods, Soni et al. (2012) did not try to optimize the bandwidth $h$, but they choose it arbitrarily to be a constant $0.15,0.19$ and 0.25 which led to similar results. In this paper, we also use the constant bandwidth.

\subsection{Confidence Interval for Quantile}

In the following, we present two theorems from Soni et al. (2012), in the first theorem they show consistency of the estimator of the quantile density function and in the other one they prove asymptotic normality of the proposed estimator.

Theorem 1. Suppose $q_{n}(u)$ given by Equation (2) is the proposed estimator of the quantile density function $q(u)$, we have

$$
\sup _{0<u<1}\left|q_{n}(u)-q(u)\right| \rightarrow 0 \quad \text { as } \quad n \rightarrow \infty .
$$

Theorem 2. Suppose $q_{n}(u)$ given by Equation (2) is the proposed estimator of the quantile density function $q(u)$. Then $\sqrt{n}\left(q_{n}(u)-q(u)\right)$ is asymptotically normal with mean zero and variance

$$
\sigma^{2}(u)=\frac{n}{(h(n))^{2}} E\left(\int_{0}^{1} d K^{*}(u, t) F_{n}\left(\hat{Q}_{n}(t)\right)\right)^{2},
$$

where $K^{*}(u, t)=K\left(\frac{t-u}{h(n)}\right) q(t)$.

Corollary 1. According to the two theorems, proposed estimator of the quantile density function (2) is a consistent estimator and asymptotically normal, therefore we can use it as a good estimator to estimating $\sigma$ in Equation (1). Hence we use the CLT and Soni's estimator of the quantile density function for constructing a CI for quantile $Q(u)$. 


\section{Simulation}

In this section, we report the results of the simulation. All studies that follow were carried out with the R statistical software package (R Core Team, 2014). We consider some wellknown distribution functions, and therefore we can easily achieve $Q(u)$. These distributions are: $\operatorname{Uniform}(0,1), \operatorname{Exponential}(1), \operatorname{Normal}(0,1), \operatorname{Lognormal}(0,1)$ and Weibull $(1,1)$. By using the simulation, samples from these distributions were generated and then by using the estimator of the quantile density function, we obtain a CI from this sample. By repeating this process, we compute $\mathrm{CP}, \mathrm{MSE}$ and the expected length of $\mathrm{CI}$. Also, we obtain CI by sectioning and jackknife methods and we compute their CP, MSE and the expected length similarly.

In the simulation, we use the samples size $n=50,100,200$ and 1000 at the level $\alpha=0.05$. For finding the estimator of the quantile density function, we consider Epanechnikov kernel and reporting the results for $h(n)=0.15$ (As mentioned, in this paper we are not trying to optimize the bandwidth $h(n)$ and we just want to show that even with constant bandwidth, the efficiency of this method is desirable). To determine CP, MSE and the expected length of CI, we generate 10000 samples of sizes 50, 100, 200 and 1000 from the specified distributions. We have taken the number of sections 10 in sectioning and jackknife methods. It is obvious that the best approach is the one providing the $\mathrm{CP}$ nearest to the nominal value (0.95, in this case) and have the shortest length. Results are reported in Tables 1-20.

We choose sample size $n=50$ in Tables 1-5. As seen in tables for $u=0.5$, the sectioning approach has more CP than jackknife and nonparametric methods, but in some cases, MSE of this method is more than the two other methods and usually the length of CI of sectioning method is more than nonparametric approach. However for $u=0.1,0.25,0.75,0.9$ results of sectioning method is not satisfactory. In almost all distributions (except lognormal distribution for $u=0.9$ ) CP of the nonparametric method is better than sectioning and jackknife methods, also MSE of this method is low and CI have the shortest length. Even in some cases for $u=0.05$ or 0.95 , the performance of the nonparametric method is better than the two other methods. As described in Section 2.2, in sectioning method, $\mathrm{m}$ should be relatively small and the number of observations $\mathrm{k}$ per section should be very large. When sample size is 50 , with choosing $m=10$, the number of observations per section is equal to 5 , where is very small, hence CI is violated in extreme quantiles. It should be noted that, as expressed in Section 2.3, we applied the jackknife approach to reducing the bias of sectioning method (where the simulation results show this), but the $\mathrm{CP}$ of sectioning method may be better than other approaches, as quantiles near the median.

As the sample size increases to 100 (Tables 6-10) or 200 (Tables 11-15), results of nonparametric method improve in center and tails (especially in the lower tail), such that for $u=0.05,0.95$ in most cases the nonparametric method outperforms the two other methods.

In Tables 16-20, we report results for the sample size 1000. By comparison CP, MSE and the length of CI in various methods we conclude, except in tails, the nonparametric 
method has good results and in almost all cases CP, MSE and the expected length of CI in this method is better than sectioning and jackknife methods.

\section{Conclusion}

According to the simulation results, for small sample size, in estimation of CI for the median, sectioning method has better performance, and in tails the results of jackknife method is favorable. But for other quantiles, the nonparametric method has higher CP (in this work, close to 0.95), lower MSE and the shortest length in comparison with the two other methods. As the sample size increases, results of nonparametric method improve in center and tails (especially in the lower tail).

We emphasize that the results of nonparametric method have been achieved for constant bandwidth and we expect that the performance of this method increase by using optimal bandwidth.

\section{Acknowledgments}

The author would like to thank the reviewer for their valuable suggestions and comments.

\section{References}

1. Babu, G. J. (1986). Efficient estimation of the reciprocal of the density quantile function at a point. Statistics and Probability Letters, 4, 133-139.

2. Bloch, D. A. and Gastwirth, J. L. (1968). On a simple estimate of the reciprocal of the density function. Annals of Mathematical Statistics, 39, 1083-1085.

3. Bofinger, E. (1975). Estimation of a density function using order statistics. Australian Journal of Statistics, 17, 1-7.

4. Chesneau, C., Dewan, I. and Doosti, H. (2016). Nonparametric estimation of a quantile density function by wavelet methods. Computation Statistics and Data Analysis, 94, 161-174.

5. Falk, M. (1986). On the estimation of the quantile density function. Statistics and Probability Letters, 4, 69-73.

6. Jones, M. C. (1992). Estimating densities, quantiles, quantile densities and density quantiles. Annals of Institute of Statistical Mathematics, 44(4), 721-727.

7. Lewis, P. A. W. and Orav, J. (1988). Simulation Methodology for Statisticians, Operations Analysts, and Engineers, (Vol. 1). CRC press.

8. Nakayama, M. K. (2012). Confidence intervals for quantiles when applying replicated latin hypercube sampling and sectioning. Proceedings of the 2012 Autumn Simulation Conference, The Society for Modeling \& Simulation International.

9. Nelson, B. L. (1990). Control variate remedies. Operations Research, 38(6), 974992. 
10. Parzen, E. (1979). Nonparametric statistical data modeling. Journal of the American Statistical Association, 7, 105-131.

11. Prakasa Rao, B. L. S. (1983). Nonparametric functional estimation. Academic Press.

12. R Core Team. (2014). R: A language and environment for statistical computing. R Foundation for Statistical Computing, Vienna, Austria. ISBN 3-900051-07-0, URL http://www.R-project.org/.

13. Reiss, R. D. (1978). Approximate distribution of the maximum deviation of histograms. Metrika, 25(1), 9-26.

14. Serfling, R. J. (1980). Approximation theorems of mathematical statistics. New York: Wiley.

15. Sheather, S. J. and Maritz, J. S. (1983). An estimate of the asymptotic standard error of the sample median. Australian Journal of Statistics, 25(1), 109-122.

16. Siddiqui, M. M. (1960). Distribution of quantitles in samples from a bivariate population. Journal of Research of the National Bureau of Standards, Section B, 64, 145-150.

17. Soni, P. and Dewan, I. and Jain, K. (2012). Nonparametric estimation of quantile density function. Computational Statistics and Data Analysis, 56, 3876-3886.

18. Steitnberg, S. M. (1983). Confidence intervals for functions of quantiles using linear combinations of order statistics. Ph. D. Thesis, University of North Carolina, Chapel Hill.

19. Tukey, J. W. (1965). Which part of the sample contains the information? Proceedings of the Mathemetical Academy of Science USA, 53, 127-134.

20. Wand, M. P. and Jones, M. C. (1995). Kernel smoothing; Monographs on statistics and applied probability. Chapman \& Hall.

21. Welsh, A. H. (1988). Asymptotically efficient estimation of the sparsity function at a point. Statistics and Probability Letters, 6, 427-432.

22. Woodruff, R. S. (1952). Confidence intervals for medians and other positional measures. Journal of the American Statistical Association, 47, 635-646. 


\section{Appendix}

Table 1: CP, MSE and the length of CI for Various Choices of $u$ in sampling from Uniform(0,1) Distribution $(\mathbf{n}=50$, replicate $=10000, \alpha=0.05)$.

\begin{tabular}{l|l|ccccccccc}
\hline \multirow{2}{*}{ Method } & Index & 0.01 & 0.05 & 0.1 & 0.25 & 0.5 & 0.75 & 0.9 & 0.95 & 0.99 \\
\hline \multirow{3}{*}{ Nonparametric } & CP & 0.6001 & 0.8722 & 0.9615 & 0.9346 & 0.9299 & 0.9252 & 0.9599 & 0.8963 & 0.6484 \\
& MSE & 0.0005 & 0.0011 & 0.0024 & 0.0037 & 0.0048 & 0.0037 & 0.0025 & 0.0013 & 0.0005 \\
& Length & 0.0393 & 0.1117 & 0.1818 & 0.2357 & 0.2707 & 0.2342 & 0.1855 & 0.1214 & 0.0455 \\
\hline \multirow{3}{*}{ Sectioning } & CP & 0.0085 & 0.0346 & 0.1735 & 0.7797 & 0.9475 & 0.7839 & 0.1663 & 0.0364 & 0.0081 \\
& MSE & 0.0285 & 0.0245 & 0.0198 & 0.0103 & 0.0036 & 0.0102 & 0.0199 & 0.0243 & 0.0286 \\
& Length & 0.1733 & 0.1714 & 0.1752 & 0.2206 & 0.2344 & 0.2207 & 0.1752 & 0.1714 & 0.1733 \\
\hline \multirow{3}{*}{ Jackknife } & CP & 0.8727 & 0.8119 & 0.6977 & 0.8201 & 0.8367 & 0.8231 & 0.7011 & 0.8127 & 0.8734 \\
& MSE & 0.0007 & 0.0017 & 0.0026 & 0.0053 & 0.0067 & 0.0054 & 0.0026 & 0.0017 & 0.0007 \\
& Length & 0.1026 & 0.1634 & 0.1979 & 0.2848 & 0.3212 & 0.2875 & 0.1981 & 0.1633 & 0.1032 \\
\hline
\end{tabular}

Table 2: CP, MSE and the length of CI for Various Choices of $u$ in sampling from $\operatorname{Exp}(1)$ Distribution $(n=50$, replicate $=10000, \alpha=0.05)$.

\begin{tabular}{|c|c|c|c|c|c|c|c|c|c|c|}
\hline Method & & 0.01 & 0.05 & 0.1 & 0.25 & 0.5 & 0.75 & 0.9 & 0.95 & 0.99 \\
\hline \multirow{3}{*}{ Nonparametric } & $\mathrm{CP}$ & 0.6289 & 0.8861 & 0.9611 & 0.9278 & 0.9175 & 0.8914 & 0.7374 & 0.4107 & 0.0736 \\
\hline & MSE & 0.0005 & 0.0014 & 0.0031 & 0.0069 & 0.0194 & 0.0475 & 0.0643 & 0.0483 & 0.3823 \\
\hline & Length & 0.0433 & 0.1242 & 0.2070 & 0.3187 & 0.5441 & 0.8534 & 0.9596 & 0.6436 & 0.2445 \\
\hline \multirow{3}{*}{ Sectioning } & $\mathrm{CP}$ & 0.0408 & 0.0611 & 0.1854 & 0.7739 & 0.9464 & 0.8672 & 0.6695 & 0.3160 & 0.0100 \\
\hline & MSE & 0.0441 & 0.0433 & 0.0430 & 0.0369 & 0.0298 & 0.0574 & 0.2584 & 0.9426 & 5.7174 \\
\hline & Length & 0.2490 & 0.2545 & 0.2776 & 0.3982 & 0.5743 & 0.8399 & 1.1305 & 1.3007 & 1.4608 \\
\hline \multirow{3}{*}{ Jackknife } & $\mathrm{CP}$ & 0.8779 & 0.8168 & 0.7269 & 0.8176 & 0.8417 & 0.8152 & 0.6784 & 0.7583 & 0.7210 \\
\hline & MSE & 0.0008 & 0.0022 & 0.0035 & 0.0100 & 0.0279 & 0.0851 & 0.2031 & 0.4013 & 0.9157 \\
\hline & Length & 0.1099 & 0.1827 & 0.2329 & 0.3928 & 0.6550 & 1.1432 & 1.7632 & 2.4787 & 3.7280 \\
\hline
\end{tabular}

Table 3: CP, MSE and the length of CI for Various Choices of $u$ in sampling from $\operatorname{Normal}(0,1)$ Distribution $(\mathbf{n}=50$, replicate $=10000, \alpha=0.05)$.

\begin{tabular}{l|l|lllllllll}
\hline \multirow{2}{*}{ Method } & Index & 0.01 & 0.05 & 0.1 & 0.25 & 0.5 & 0.75 & 0.9 & 0.95 & 0.99 \\
\hline \multirow{3}{*}{ Nonparametric } & CP & 0.1363 & 0.5713 & 0.8610 & 0.9285 & 0.9228 & 0.9043 & 0.8516 & 0.6117 & 0.1529 \\
& MSE & 0.0737 & 0.0235 & 0.0395 & 0.0360 & 0.0296 & 0.0327 & 0.0398 & 0.0251 & 0.0754 \\
& Length & 0.1742 & 0.4814 & 0.7510 & 0.7399 & 0.6740 & 0.7032 & 0.7522 & 0.5173 & 0.1990 \\
\hline \multirow{3}{*}{ Sectioning } & CP & 0.0025 & 0.1927 & 0.5051 & 0.8445 & 0.9488 & 0.8520 & 0.5086 & 0.1941 & 0.0036 \\
& MSE & 1.4592 & 0.4186 & 0.1820 & 0.0633 & 0.0284 & 0.0625 & 0.1793 & 0.4179 & 1.4544 \\
& Length & 0.8142 & 0.7561 & 0.7002 & 0.6902 & 0.6610 & 0.6930 & 0.7004 & 0.7563 & 0.8137 \\
\hline \multirow{3}{*}{ Jackknife } & CP & 0.7512 & 0.7837 & 0.6923 & 0.8254 & 0.8424 & 0.8176 & 0.6951 & 0.7774 & 0.7554 \\
& MSE & 0.1517 & 0.1015 & 0.0676 & 0.0519 & 0.0433 & 0.0522 & 0.0676 & 0.1014 & 0.1487 \\
& Length & 1.5143 & 1.2477 & 1.0185 & 0.8927 & 0.8160 & 0.8958 & 1.0193 & 1.2472 & 1.4991 \\
\hline
\end{tabular}


Table 4: CP, MSE and the length of CI for Various Choices of $u$ in sampling from Lognormal $(0,1)$ Distribution $(\mathbf{n}=50$, replicate $=10000, \alpha=0.05)$.

\begin{tabular}{|c|c|c|c|c|c|c|c|c|c|c|}
\hline Method & Index & 0.01 & 0.05 & 0.1 & 0.25 & 0.5 & 0.75 & 0.9 & 0.95 & 0.99 \\
\hline \multirow{3}{*}{ Nonparametric } & $\mathrm{CP}$ & 0.4176 & 0.8382 & 0.9502 & 0.9335 & 0.9128 & 0.8479 & 0.4995 & 0.2100 & 0.0232 \\
\hline & MSE & 0.0021 & 0.0028 & 0.0059 & 0.0102 & 0.0298 & 0.0851 & 0.0969 & 0.0980 & 2.2224 \\
\hline & Length & 0.0650 & 0.1783 & 0.2867 & 0.3890 & 0.6740 & 1.1437 & 1.1757 & 0.7661 & 0.2825 \\
\hline \multirow{3}{*}{ Sectioning } & $\mathrm{CP}$ & 0.0151 & 0.0861 & 0.2559 & 0.7916 & 0.9405 & 0.8920 & 0.7781 & 0.4626 & 0.0464 \\
\hline & MSE & 0.0969 & 0.0733 & 0.0658 & 0.0580 & 0.0697 & 0.1469 & 0.7388 & 3.2679 & 40.377 \\
\hline & Length & 0.3252 & 0.3317 & 0.3601 & 0.5182 & 0.8320 & 1.4925 & 2.9955 & 3.7199 & 4.1655 \\
\hline \multirow{3}{*}{ Jackknife } & $\mathrm{CP}$ & 0.8249 & 0.8158 & 0.7215 & 0.8260 & 0.8363 & 0.8084 & 0.6654 & 0.7450 & 0.6716 \\
\hline & MSE & 0.0031 & 0.0052 & 0.0063 & 0.0152 & 0.0464 & 0.2122 & 0.9051 & 2.8640 & 15.912 \\
\hline & Length & 0.2166 & 0.2834 & 0.3116 & 0.4840 & 0.8441 & 1.8055 & 3.7060 & 6.6068 & 15.636 \\
\hline
\end{tabular}

Table 5: CP, MSE and the length of CI for Various Choices of $u$ in sampling from Weibull $(1,1)$ Distribution $(\mathbf{n}=50$, replicate $=10000, \alpha=0.05)$.

\begin{tabular}{|c|c|c|c|c|c|c|c|c|c|c|}
\hline Method & Index & 0.01 & 0.05 & 0.1 & 0.25 & 0.5 & 0.75 & 0.9 & 0.95 & 0.99 \\
\hline \multirow{3}{*}{ Nonparametric } & $\mathrm{CP}$ & 0.6028 & 0.8849 & 0.9596 & 0.9306 & 0.9138 & 0.8894 & 0.7395 & 0.4113 & 0.0759 \\
\hline & MSE & 0.0005 & 0.0014 & 0.0032 & 0.0069 & 0.0194 & 0.0475 & 0.0639 & 0.0473 & 0.3659 \\
\hline & Length & 0.0432 & 0.1236 & 0.2070 & 0.3187 & 0.5426 & 0.8545 & 0.9569 & 0.6465 & 0.2445 \\
\hline \multirow{3}{*}{ Sectioning } & $\mathrm{CP}$ & 0.0369 & 0.0644 & 0.1785 & 0.7753 & 0.9457 & 0.8755 & 0.6686 & 0.3235 & 0.0104 \\
\hline & MSE & 0.0443 & 0.0436 & 0.0430 & 0.0367 & 0.0297 & 0.0572 & 0.2587 & 0.9404 & 5.7220 \\
\hline & Length & 0.2486 & 0.2557 & 0.2768 & 0.3960 & 0.5743 & 0.8448 & 1.1212 & 1.3101 & 1.4562 \\
\hline \multirow{3}{*}{ Jackknife } & $\mathrm{CP}$ & 0.8812 & 0.8107 & 0.7108 & 0.8203 & 0.8382 & 0.8122 & 0.6791 & 0.7611 & 0.7204 \\
\hline & MSE & 0.0008 & 0.0021 & 0.0035 & 0.0105 & 0.0283 & 0.0835 & 0.2019 & 0.4161 & 0.9365 \\
\hline & Length & 0.1092 & 0.1789 & 0.2318 & 0.4024 & 0.6589 & 1.1319 & 1.7579 & 2.5219 & 3.7683 \\
\hline
\end{tabular}

Table 6: CP, MSE and the length of CI for Various Choices of $u$ in sampling from Uniform $(0,1)$ Distribution $(n=100$, replicate $=10000, \alpha=0.05)$.

\begin{tabular}{|c|c|c|c|c|c|c|c|c|c|c|}
\hline Method & Index & 0.01 & 0.05 & 0.1 & 0.25 & 0.5 & 0.75 & 0.9 & 0.95 & 0.99 \\
\hline \multirow{3}{*}{ Nonparametric } & $\mathrm{CP}$ & 0.6978 & 0.8973 & 0.9651 & 0.9420 & 0.9411 & 0.9400 & 0.9676 & 0.9113 & 0.7188 \\
\hline & MSE & 0.0001 & 0.0005 & 0.0011 & 0.0019 & 0.0024 & 0.0019 & 0.0012 & 0.0005 & 0.0002 \\
\hline & Length & 0.0286 & 0.0799 & 0.1287 & 0.1681 & 0.1935 & 0.1680 & 0.1302 & 0.0834 & 0.0308 \\
\hline \multirow{3}{*}{ Sectioning } & $\mathrm{CP}$ & 0.0214 & 0.1533 & 0.5356 & 0.8626 & 0.9465 & 0.8652 & 0.5373 & 0.1495 & 0.0213 \\
\hline & MSE & 0.0087 & 0.0074 & 0.0065 & 0.0037 & 0.0019 & 0.0037 & 0.0064 & 0.0074 & 0.0087 \\
\hline & Length & 0.1025 & 0.1082 & 0.1317 & 0.1562 & 0.1707 & 0.1565 & 0.1310 & 0.1080 & 0.1022 \\
\hline \multirow{3}{*}{ Jackknife } & $\mathrm{CP}$ & 0.7100 & 0.6962 & 0.7856 & 0.8297 & 0.8423 & 0.8192 & 0.7757 & 0.7006 & 0.7133 \\
\hline & MSE & 0.0003 & 0.0007 & 0.0013 & 0.0024 & 0.0030 & 0.0023 & 0.0013 & 0.0007 & 0.0003 \\
\hline & Length & 0.0689 & 0.1038 & 0.1414 & 0.1903 & 0.2135 & 0.1891 & 0.1410 & 0.1058 & 0.0698 \\
\hline
\end{tabular}


Table 7: CP, MSE and the length of CI for Various Choices of $u$ in sampling from $\operatorname{Exp}(1)$ Distribution $(n=100$, replicate $=10000, \alpha=0.05)$.

\begin{tabular}{|c|c|c|c|c|c|c|c|c|c|c|}
\hline Met & Index & 0.01 & 0.05 & 0.1 & 0.25 & 0.5 & 0.75 & 0.9 & 0.95 & 0.99 \\
\hline \multirow{3}{*}{ Nonparametric } & $\mathrm{CP}$ & 0.7132 & 0.9063 & 0.9663 & 0.9385 & 0.9360 & 0.9345 & 0.8811 & 0.5594 & 0.1054 \\
\hline & MSE & 0.0002 & 0.0006 & 0.0015 & 0.0034 & 0.0100 & 0.0302 & 0.0621 & 0.0375 & 0.1781 \\
\hline & Length & 0.0312 & 0.0883 & 0.1454 & 0.2273 & 0.3920 & 0.6808 & 0.9665 & 0.6830 & 0.2665 \\
\hline \multirow{3}{*}{ Sectioning } & $\mathrm{CP}$ & 0.0432 & 0.1697 & 0.5495 & 0.8433 & 0.9480 & 0.9206 & 0.7537 & 0.5906 & 0.0565 \\
\hline & MSE & 0.0110 & 0.0112 & 0.0108 & & 0.0122 & 0.0267 & & & 3.2361 \\
\hline & Length & 0.1250 & 0.1393 & 0.1746 & 0.2474 & 0.3848 & 0.6208 & 0.9278 & 1.1441 & 1.4566 \\
\hline \multirow{3}{*}{ Jackknife } & $\mathrm{CP}$ & 0.7166 & 0.6991 & 0.7888 & 0.8211 & 0.8396 & 0.8288 & 0.7686 & 0.6622 & 0.5796 \\
\hline & MSE & 0.0003 & 0.0008 & 0.0017 & 0.0044 & 0.0122 & 0.0367 & 0.1166 & 0.2125 & 0.9036 \\
\hline & Length & 0.0723 & 0.1127 & 0.1633 & 0.2605 & 0.4324 & 0.7511 & 1.3386 & 1.7934 & 3.4441 \\
\hline
\end{tabular}

Table 8: CP, MSE and the length of CI for Various Choices of $u$ in sampling from Normal $(0,1)$ Distribution $(n=100$, replicate $=10000, \alpha=0.05)$.

\begin{tabular}{|c|c|c|c|c|c|c|c|c|c|c|}
\hline Method & Index & 0.01 & 0.05 & 0.1 & 0.25 & 0.5 & 0.75 & 0.9 & 0.95 & 0.99 \\
\hline \multirow{3}{*}{ Nonparametric } & $\mathrm{CP}$ & 0.1931 & 0.7234 & 0.9467 & 0.9459 & 0.9389 & 0.9359 & 0.9456 & 0.7459 & 0.1990 \\
\hline & MSE & 0.0314 & 0.0171 & 0.0313 & 0.0197 & 0.0156 & 0.0188 & 0.0304 & 0.0175 & 0.0316 \\
\hline & Length & 0.1832 & 0.4759 & 0.6852 & 0.5491 & 0.4901 & 0.5362 & 0.6771 & 0.4869 & 0.1938 \\
\hline \multirow{3}{*}{ Sectioning } & $\mathrm{CP}$ & 0.0267 & 0.4620 & 0.6929 & 0.8990 & 0.9516 & 0.8941 & 0.6900 & 0.4715 & 0.0269 \\
\hline & MSE & 0.7267 & 0.1443 & 0.0716 & 0.0233 & 0.0139 & 0.0240 & 0.0723 & 0.1432 & 0.7364 \\
\hline & Length & 0.6964 & 0.5965 & 0.5639 & 0.4938 & 0.4622 & 0.4928 & 0.5658 & 0.5975 & 0.6950 \\
\hline \multirow{3}{*}{ Jackknife } & $\mathrm{CP}$ & 0.6095 & 0.6743 & 0.7860 & 0.8206 & 0.8462 & 0.8296 & 0.7783 & 0.6739 & 0.6075 \\
\hline & MSE & 0.1318 & 0.0507 & 0.0384 & 0.0233 & 0.0189 & 0.0233 & 0.0387 & 0.0507 & 0.1323 \\
\hline & Length & 1.3467 & 0.8804 & 0.7679 & 0.5983 & 0.5388 & 0.5977 & 0.7710 & 0.8814 & 1.3560 \\
\hline
\end{tabular}

Table 9: CP, MSE and the length of CI for Various Choices of $u$ in sampling from Lognormal $(0,1)$ Distribution $(n=100$, replicate $=10000, \alpha=0.05)$.

\begin{tabular}{|c|c|c|c|c|c|c|c|c|c|c|}
\hline Method & Index & 0.01 & 0.05 & 0.1 & 0.25 & 0.5 & 0.75 & 0.9 & 0.95 & 0.99 \\
\hline \multirow{3}{*}{ Nonparametric } & $\mathrm{CP}$ & 0.4481 & 0.8672 & 0.9640 & 0.9430 & 0.9393 & 0.9251 & 0.7201 & 0.3229 & 0.0340 \\
\hline & MSE & 0.0007 & 0.0013 & 0.0029 & 0.0051 & 0.0161 & 0.0672 & 0.1182 & 0.0770 & 1.3345 \\
\hline & Length & 0.0487 & 0.1319 & 0.2055 & 0.2791 & 0.4960 & 1.0163 & 1.3342 & 0.9051 & 0.3417 \\
\hline \multirow{3}{*}{ Sectioning } & $\mathrm{CP}$ & 0.0130 & 0.2511 & 0.5834 & 0.8466 & 0.9427 & 0.9278 & 0.8257 & 0.7261 & 0.1317 \\
\hline & MSE & 0.0303 & 0.0187 & 0.0167 & 0.0165 & 0.0251 & 0.0714 & 0.3698 & 1.2822 & 25.193 \\
\hline & Length & 0.1764 & 0.1861 & 0.2233 & 0.3065 & 0.5189 & 1.0470 & 2.0936 & 3.4594 & 4.8413 \\
\hline \multirow{3}{*}{ Jackknife } & $\mathrm{CP}$ & 0.6684 & 0.6947 & 0.7828 & 0.8271 & 0.8484 & 0.8163 & 0.7629 & 0.6519 & 0.5288 \\
\hline & MSE & 0.0019 & 0.0022 & 0.0032 & 0.0063 & 0.0195 & 0.0904 & 0.5000 & 1.3808 & 15.718 \\
\hline & Length & 0.1689 & 0.1857 & 0.2224 & 0.3123 & 0.5468 & 1.1788 & 2.7717 & 4.5789 & 13.605 \\
\hline
\end{tabular}


Table 10: CP, MSE and the length of $\mathrm{CI}$ for Various Choices of $\mathbf{u}$ in sampling from Weibull $(1,1)$ Distribution $(n=100$, replicate $=10000, \alpha=0.05)$.

\begin{tabular}{|c|c|c|c|c|c|c|c|c|c|c|}
\hline Method & Index & 0.01 & 0.05 & 0.1 & 0.25 & 0.5 & 0.75 & 0.9 & 0.95 & 0.99 \\
\hline \multirow{3}{*}{ Nonparametric } & $\mathrm{CP}$ & 0.7238 & 0.9156 & 0.9667 & 0.9366 & 0.9317 & 0.9345 & 0.8849 & 0.5541 & 0.1071 \\
\hline & MSE & 0.0002 & 0.0006 & 0.0015 & 0.0034 & 0.0100 & 0.0304 & 0.0620 & 0.0382 & 0.1737 \\
\hline & Length & 0.0311 & 0.0880 & 0.1451 & 0.2267 & 0.3919 & 0.6831 & 0.9677 & 0.6820 & 0.2667 \\
\hline \multirow{3}{*}{ Sectioning } & $\mathrm{CP}$ & 0.0427 & 0.1712 & 0.5345 & 0.8410 & 0.9431 & 0.9210 & 0.7503 & 0.5819 & 0.0524 \\
\hline & MSE & 0.0111 & 0.0110 & 0.0110 & 0.0109 & 0.0124 & 0.0266 & 0.1306 & 0.3535 & 3.2660 \\
\hline & Length & 0.1256 & 0.1388 & 0.1749 & 0.2453 & 0.3844 & 0.6202 & 0.9276 & 1.1349 & 1.4512 \\
\hline \multirow{3}{*}{ Jackknife } & $\mathrm{CP}$ & 0.7219 & 0.7029 & 0.7855 & 0.8276 & 0.8419 & 0.8314 & 0.7781 & 0.6688 & 0.5788 \\
\hline & MSE & 0.0003 & 0.0008 & 0.0017 & 0.0044 & 0.0122 & 0.0375 & 0.1195 & 0.2124 & 0.8850 \\
\hline & Length & 0.0725 & 0.1119 & 0.1629 & 0.2586 & 0.4321 & 0.7587 & 1.3552 & 1.8002 & 3.4177 \\
\hline
\end{tabular}

Table 11: CP, MSE and the length of CI for Various Choices of $u$ in sampling from Uniform(0,1) Distribution $(\mathbf{n}=\mathbf{2 0 0}$, replicate $=10000, \alpha=0.05)$.

\begin{tabular}{|c|c|c|c|c|c|c|c|c|c|c|}
\hline Method & Index & 0.01 & 0.05 & 0.1 & 0.25 & 0.5 & 0.75 & 0.9 & 0.95 & 0.99 \\
\hline \multirow{3}{*}{ Nonparametric } & $\mathrm{CP}$ & 0.7691 & 0.9174 & 0.9629 & 0.9469 & 0.9427 & 0.9433 & 0.9670 & 0.9225 & 0.7738 \\
\hline & MSE & 0.0001 & 0.0002 & 0.0006 & 0.0009 & 0.0012 & 0.0009 & 0.0006 & 0.0002 & 0.0001 \\
\hline & Length & 0.0204 & 0.0567 & 0.0908 & 0.1198 & 0.1378 & 0.1197 & 0.0914 & 0.0580 & 0.0212 \\
\hline \multirow{3}{*}{ Sectioning } & $\mathrm{CP}$ & 0.0431 & 0.5081 & 0.7378 & 0.9086 & 0.9505 & 0.9101 & 0.7288 & 0.5051 & 0.0404 \\
\hline & MSE & 0.0024 & 0.0022 & 0.0020 & 0.0014 & 0.0011 & 0.0014 & 0.0020 & 0.0022 & 0.0024 \\
\hline & Length & 0.0567 & 0.0761 & 0.0895 & 0.1150 & 0.1287 & 0.1153 & 0.0897 & 0.0752 & 0.0565 \\
\hline \multirow{3}{*}{ Jackknife } & $\mathrm{CP}$ & 0.6870 & 0.7797 & 0.8103 & 0.8434 & 0.8603 & 0.8489 & 0.8080 & 0.7736 & 0.6927 \\
\hline & MSE & 0.0001 & 0.0004 & 0.0006 & 0.0011 & 0.0015 & 0.0011 & 0.0006 & 0.0004 & 0.0001 \\
\hline & Length & 0.0405 & 0.0748 & 0.0969 & 0.1306 & 0.1494 & 0.1316 & 0.0960 & 0.0746 & 0.0407 \\
\hline
\end{tabular}

Table 12: CP, MSE and the length of $\mathrm{CI}$ for Various Choices of $\mathrm{u}$ in sampling from $\operatorname{Exp}(1)$ Distribution $(n=200$, replicate $=10000, \alpha=0.05)$.

\begin{tabular}{|c|c|c|c|c|c|c|c|c|c|c|}
\hline Method & Index & 0.01 & 0.05 & 0.1 & 0.25 & 0.5 & 0.75 & 0.9 & 0.95 & 0.99 \\
\hline \multirow{3}{*}{ Nonparametric } & $\mathrm{CP}$ & 0.7841 & 0.9279 & 0.9677 & 0.9437 & 0.9432 & 0.9538 & 0.9567 & 0.7153 & 0.1538 \\
\hline & MSE & 0.0001 & 0.0003 & 0.0007 & 0.0017 & 0.0051 & 0.0165 & 0.0518 & 0.0306 & 0.0483 \\
\hline & Length & 0.0221 & 0.0624 & 0.1025 & 0.1610 & 0.2799 & 0.5040 & 0.8901 & 0.6657 & 0.2704 \\
\hline \multirow{3}{*}{ Sectioning } & $\mathrm{CP}$ & 0.0563 & 0.5089 & 0.7335 & 0.9018 & 0.9504 & 0.9311 & 0.8383 & 0.6968 & 0.1866 \\
\hline & MSE & 0.0028 & 0.0028 & 0.0030 & 0.0036 & 0.0056 & 0.0140 & 0.0598 & 0.1765 & 1.5697 \\
\hline & Length & 0.0635 & 0.0871 & 0.1097 & 0.1674 & 0.2756 & 0.4556 & 0.7331 & 0.9607 & 1.3786 \\
\hline \multirow{3}{*}{ Jackknife } & $\mathrm{CP}$ & 0.6935 & 0.7830 & 0.8090 & 0.8481 & 0.8623 & 0.8419 & 0.8040 & 0.7605 & 0.6058 \\
\hline & MSE & 0.0001 & 0.0004 & 0.0008 & 0.0020 & 0.0059 & 0.0179 & 0.0565 & 0.1249 & 0.4935 \\
\hline & Length & 0.0415 & 0.0800 & 0.1077 & 0.1774 & 0.3005 & 0.5245 & 0.9314 & 1.3849 & 2.6603 \\
\hline
\end{tabular}


Table 13: CP, MSE and the length of CI for Various Choices of $u$ in sampling from $\operatorname{Normal}(0,1)$ Distribution $(n=200$, replicate $=10000, \alpha=0.05)$.

\begin{tabular}{|c|c|c|c|c|c|c|c|c|c|c|}
\hline Method & Index & 0.01 & 0.05 & 0.1 & 0.25 & 0.5 & 0.75 & 0.9 & 0.95 & 0.99 \\
\hline \multirow{3}{*}{ Nonparametric } & $\mathrm{CP}$ & 0.2469 & 0.8218 & 0.9769 & 0.9557 & 0.9489 & 0.9470 & 0.9744 & 0.8370 & 0.2569 \\
\hline & MSE & 0.0106 & 0.0122 & 0.0212 & 0.0101 & 0.0080 & 0.0099 & 0.0208 & 0.0123 & 0.0109 \\
\hline & Length & 0.1698 & 0.4207 & 0.5682 & 0.3943 & 0.3513 & 0.3889 & 0.5621 & 0.4238 & 0.1736 \\
\hline \multirow{3}{*}{ Sectioning } & $\mathrm{CP}$ & 0.1297 & 0.6518 & 0.8187 & 0.9249 & 0.9502 & 0.9218 & 0.8150 & 0.6478 & 0.1309 \\
\hline & MSE & 0.3218 & 0.0612 & 0.0268 & 0.0108 & 0.0073 & 0.0106 & 0.0270 & 0.0616 & 0.3229 \\
\hline & Length & 0.5915 & 0.4935 & 0.4310 & 0.3626 & 0.3358 & 0.3615 & 0.4310 & 0.4911 & 0.5924 \\
\hline \multirow{3}{*}{ Jackknife } & $\mathrm{CP}$ & 0.6171 & 0.7737 & 0.8124 & 0.8425 & 0.8651 & 0.8498 & 0.8025 & 0.7601 & 0.6186 \\
\hline & MSE & 0.0722 & 0.0294 & 0.0187 & 0.0110 & 0.0091 & 0.0110 & 0.0184 & 0.0296 & 0.0722 \\
\hline & Length & 1.0251 & 0.6725 & 0.5353 & 0.4119 & 0.3739 & 0.4113 & 0.5323 & 0.6746 & 1.0321 \\
\hline
\end{tabular}

Table 14: CP, MSE and the length of CI for Various Choices of $u$ in sampling from $\operatorname{Lognormal}(0,1)$ Distribution $(\mathbf{n}=200$, replicate $=10000, \alpha=0.05)$.

\begin{tabular}{|c|c|c|c|c|c|c|c|c|c|c|}
\hline Method & Index & 0.01 & 0.05 & 0.1 & 0.25 & 0.5 & 0.75 & 0.9 & 0.95 & 0.99 \\
\hline \multirow{3}{*}{ Nonparametric } & $\mathrm{CP}$ & 0.4892 & 0.8930 & 0.9690 & 0.9447 & 0.9438 & 0.9511 & 0.8857 & 0.4900 & 0.0613 \\
\hline & MSE & 0.0002 & 0.0006 & 0.0014 & 0.0026 & 0.0082 & 0.0409 & 0.1262 & 0.0687 & 0.4003 \\
\hline & Length & 0.0350 & 0.0944 & 0.1462 & 0.1976 & 0.3541 & 0.7921 & 1.3900 & 1.0015 & 0.3919 \\
\hline \multirow{3}{*}{ Sectioning } & $\mathrm{CP}$ & 0.0432 & 0.5612 & 0.7638 & 0.9041 & 0.9435 & 0.9391 & 0.8636 & 0.7529 & 0.3159 \\
\hline & MSE & 0.0097 & 0.0055 & 0.0046 & 0.0051 & 0.0102 & 0.0353 & 0.1971 & 0.7507 & 12.566 \\
\hline & Length & 0.1055 & 0.1266 & 0.1435 & 0.2036 & 0.3571 & 0.7360 & 1.5649 & 2.5280 & 5.3603 \\
\hline \multirow{3}{*}{ Jackknife } & $\mathrm{CP}$ & 0.6639 & 0.7805 & 0.8098 & 0.8478 & 0.8647 & 0.8479 & 0.8054 & 0.7549 & 0.5813 \\
\hline & MSE & 0.0009 & 0.0012 & 0.0015 & 0.0029 & 0.0093 & 0.0436 & 0.2461 & 0.7921 & 8.0904 \\
\hline & Length & 0.1155 & 0.1358 & 0.1508 & 0.2110 & 0.3776 & 0.8188 & 1.9445 & 3.4881 & 10.635 \\
\hline
\end{tabular}

Table 15: CP, MSE and the length of CI for Various Choices of $u$ in sampling from Weibull $(1,1)$ Distribution $(n=200$, replicate $=10000, \alpha=0.05)$.

\begin{tabular}{|c|c|c|c|c|c|c|c|c|c|c|}
\hline Method & Index & 0.01 & 0.05 & 0.1 & 0.25 & 0.5 & 0.75 & 0.9 & 0.95 & 0.99 \\
\hline \multirow{3}{*}{ Nonparametric } & $\mathrm{CP}$ & 0.7916 & 0.9300 & 0.9696 & 0.9465 & 0.9460 & 0.9565 & 0.9552 & 0.7110 & 0.1532 \\
\hline & MSE & 0.0001 & 0.0003 & 0.0007 & 0.0017 & 0.0051 & 0.0166 & 0.0518 & 0.0309 & 0.0518 \\
\hline & Length & 0.0222 & 0.0623 & 0.1023 & 0.1608 & 0.2797 & 0.5056 & 0.8907 & 0.6658 & 0.2695 \\
\hline \multirow{3}{*}{ Sectioning } & $\mathrm{CP}$ & 0.0533 & 0.5091 & 0.7253 & 0.8959 & 0.9487 & 0.9364 & 0.8414 & 0.6931 & 0.1852 \\
\hline & MSE & 0.0028 & 0.0029 & 0.0030 & 0.0037 & 0.0056 & 0.0141 & 0.0581 & 0.1781 & 1.5710 \\
\hline & Length & 0.0628 & 0.0875 & 0.1092 & 0.1681 & 0.2755 & 0.4553 & 0.7329 & 0.9599 & 1.3819 \\
\hline \multirow{3}{*}{ Jackknife } & $\mathrm{CP}$ & 0.6856 & 0.7760 & 0.8151 & 0.8497 & 0.8613 & 0.8394 & 0.8065 & 0.7585 & 0.6079 \\
\hline & MSE & 0.0001 & 0.0004 & 0.0008 & 0.0020 & 0.0059 & 0.0180 & 0.0556 & 0.1241 & 0.4870 \\
\hline & Length & 0.0416 & 0.0794 & 0.1080 & 0.1770 & 0.2998 & 0.5261 & 0.9246 & 1.3802 & 2.6343 \\
\hline
\end{tabular}


Table 16: CP, MSE and the length of $\mathrm{CI}$ for Various Choices of $\mathbf{u}$ in sampling from $\operatorname{Uniform}(\mathbf{0 , 1})$ Distribution $(\mathbf{n}=1000$, replicate $=10000, \alpha=0.05)$.

\begin{tabular}{|c|c|c|c|c|c|c|c|c|c|c|}
\hline Method & Index & 0.01 & 0.05 & 0.1 & 0.25 & 0.5 & 0.75 & 0.9 & 0.95 & 0.99 \\
\hline \multirow{3}{*}{ Nonparametric } & $\mathrm{CP}$ & 0.8327 & 0.9319 & 0.9681 & 0.9534 & 0.9420 & 0.9405 & 0.9744 & 0.9421 & 0.8543 \\
\hline & MSE & 0.00001 & 0.00004 & 0.0001 & 0.0002 & 0.0002 & 0.0002 & 0.0001 & 0.00004 & 0.00001 \\
\hline & Length & 0.0092 & 0.0255 & 0.0405 & 0.0536 & 0.0619 & 0.0538 & 0.0407 & 0.0256 & 0.0093 \\
\hline \multirow{3}{*}{ Sectioning } & $\mathrm{CP}$ & 0.4786 & 0.8532 & 0.9117 & 0.9391 & 0.9489 & 0.9439 & 0.9094 & 0.8532 & 0.4839 \\
\hline & MSE & 0.00011 & 0.00013 & 0.0002 & 0.0002 & 0.0002 & 0.0002 & 0.0002 & 0.00013 & 0.00011 \\
\hline & Length & 0.0170 & 0.0288 & 0.0379 & 0.0531 & 0.0610 & 0.0533 & 0.0379 & 0.0288 & 0.0169 \\
\hline \multirow{3}{*}{ Jackknife } & $\mathrm{CP}$ & 0.7693 & 0.8444 & 0.8685 & 0.8951 & 0.8993 & 0.8942 & 0.8614 & 0.8443 & 0.7751 \\
\hline & MSE & 0.00002 & 0.00006 & 0.0001 & 0.0002 & 0.0003 & 0.0002 & 0.0001 & 0.00006 & 0.00002 \\
\hline & Length & 0.0155 & 0.0299 & 0.0404 & 0.0565 & 0.0644 & 0.0564 & 0.0400 & 0.0301 & 0.0156 \\
\hline
\end{tabular}

Table 17: CP, MSE and the length of CI for Various Choices of $u$ in sampling from $\operatorname{Exp}(1)$ Distribution $(\mathbf{n}=1000$, replicate $=10000, \alpha=0.05)$.

\begin{tabular}{|c|c|c|c|c|c|c|c|c|c|c|}
\hline Method & Index & 0.01 & 0.05 & 0.1 & 0.25 & 0.5 & 0.75 & 0.9 & 0.95 & 0.99 \\
\hline \multirow{3}{*}{ Nonparametric } & $\mathrm{CP}$ & 0.8772 & 0.9475 & 0.9516 & 0.9440 & 0.9520 & 0.9512 & 0.9593 & 0.9224 & 0.2692 \\
\hline & MSE & 0.00001 & 0.00005 & 0.0001 & 0.0003 & 0.0010 & 0.0035 & 0.0228 & 0.0159 & 0.0056 \\
\hline & Length & 0.0100 & 0.0279 & 0.0456 & 0.0721 & 0.1255 & 0.2316 & 0.5920 & 0.4921 & 0.2145 \\
\hline \multirow{3}{*}{ Sectioning } & $\mathrm{CP}$ & 0.4829 & 0.8577 & 0.9109 & 0.9415 & 0.9523 & 0.9427 & 0.9268 & 0.8940 & 0.6379 \\
\hline & MSE & 0.0001 & 0.0002 & 0.0002 & 0.0004 & 0.0010 & 0.0030 & 0.0096 & 0.0238 & 0.2325 \\
\hline & Length & 0.0174 & 0.0309 & 0.0430 & 0.0722 & 0.1242 & 0.2118 & 0.3613 & 0.5152 & 0.9896 \\
\hline \multirow{3}{*}{ Jackknife } & $\mathrm{CP}$ & 0.7693 & 0.8420 & 0.8664 & 0.8920 & 0.9003 & 0.8911 & 0.8670 & 0.8389 & 0.7522 \\
\hline & MSE & 0.00002 & 0.00007 & 0.0001 & 0.0004 & 0.0011 & 0.0033 & 0.0103 & 0.0228 & 0.1300 \\
\hline & Length & 0.0159 & 0.0317 & 0.0450 & 0.0757 & 0.1291 & 0.2250 & 0.3983 & 0.5919 & 1.4131 \\
\hline
\end{tabular}

Table 18: CP, MSE and the length of CI for Various Choices of $u$ in sampling from Normal $(0,1)$ Distribution $(\mathbf{n}=1000$, replicate $=10000, \alpha=0.05)$.

\begin{tabular}{|c|c|c|c|c|c|c|c|c|c|c|}
\hline Method & Index & 0.01 & 0.05 & 0.1 & 0.25 & 0.5 & 0.75 & 0.9 & 0.95 & 0.99 \\
\hline \multirow{3}{*}{ Nonparametric } & $\mathrm{CP}$ & 0.3791 & 0.9437 & 0.9597 & 0.9519 & 0.9520 & 0.9617 & 0.9428 & 0.9474 & 0.3783 \\
\hline & MSE & 0.0011 & 0.0044 & 0.0066 & 0.0020 & 0.0016 & 0.0020 & 0.0066 & 0.0044 & 0.0010 \\
\hline & Length & 0.1120 & 0.2592 & 0.3194 & 0.1774 & 0.1576 & 0.1769 & 0.3183 & 0.2603 & 0.1117 \\
\hline \multirow{3}{*}{ Sectioning } & $\mathrm{CP}$ & 0.6065 & 0.8784 & 0.9237 & 0.9472 & 0.9474 & 0.9457 & 0.9219 & 0.8882 & 0.6155 \\
\hline & MSE & 0.0403 & 0.0065 & 0.0035 & 0.0019 & 0.0016 & 0.0019 & 0.0035 & 0.0066 & 0.0402 \\
\hline & Length & 0.3827 & 0.2512 & 0.2080 & 0.1671 & 0.1543 & 0.1677 & 0.2081 & 0.2521 & 0.3842 \\
\hline \multirow{3}{*}{ Jackknife } & $\mathrm{CP}$ & 0.7580 & 0.8420 & 0.8645 & 0.8838 & 0.9054 & 0.8910 & 0.8682 & 0.8405 & 0.7593 \\
\hline & MSE & 0.0187 & 0.0052 & 0.0033 & 0.0021 & 0.0017 & 0.0021 & 0.0034 & 0.0054 & 0.0187 \\
\hline & Length & 0.5358 & 0.2839 & 0.2249 & 0.1782 & 0.1633 & 0.1784 & 0.2282 & 0.2868 & 0.5364 \\
\hline
\end{tabular}


Table 19: CP, MSE and the length of CI for Various Choices of $u$ in sampling from Lognormal $(0,1)$ Distribution $(n=1000$, replicate $=10000, \alpha=0.05)$.

\begin{tabular}{l|l|ccccccccc}
\hline \multirow{2}{*}{ Method } & Index & 0.01 & 0.05 & 0.1 & 0.25 & 0.5 & 0.75 & 0.9 & 0.95 & 0.99 \\
\hline \multirow{3}{*}{ Nonparametric } & CP & 0.5124 & 0.8903 & 0.9547 & 0.9582 & 0.9601 & 0.9518 & 0.9417 & 0.8451 & 0.1840 \\
& MSE & 0.00002 & 0.0001 & 0.0003 & 0.0005 & 0.0017 & 0.0090 & 0.0894 & 0.0613 & 0.0422 \\
& Length & 0.0159 & 0.0426 & 0.0655 & 0.0884 & 0.1595 & 0.3716 & 1.1719 & 0.9685 & 0.4159 \\
\hline \multirow{3}{*}{ Sectioning } & CP & 0.5597 & 0.8691 & 0.9206 & 0.9413 & 0.9541 & 0.9428 & 0.9299 & 0.9003 & 0.6887 \\
& MSE & 0.0007 & 0.0004 & 0.0004 & 0.0006 & 0.0017 & 0.0071 & 0.0384 & 0.1317 & 2.3786 \\
& Length & 0.0448 & 0.0516 & 0.0596 & 0.0872 & 0.1564 & 0.3309 & 0.7473 & 1.3006 & 3.8243 \\
\hline \multirow{3}{*}{ Jackknife } & CP & 0.7645 & 0.8459 & 0.8698 & 0.8951 & 0.9002 & 0.8885 & 0.8661 & 0.8397 & 0.7488 \\
& MSE & 0.0002 & 0.0002 & 0.0003 & 0.0005 & 0.0017 & 0.0079 & 0.0436 & 0.1451 & 1.9471 \\
& Length & 0.0537 & 0.0560 & 0.0636 & 0.0903 & 0.1625 & 0.3490 & 0.8183 & 1.4929 & 5.4697 \\
\hline
\end{tabular}

Table 20: CP, MSE and the length of CI for Various Choices of $\mathrm{u}$ in sampling from Weibull $(1,1)$ Distribution $(\mathbf{n}=1000$, replicate $=10000, \alpha=0.05)$.

\begin{tabular}{l|l|ccccccccc}
\hline Method & Index & 0.01 & 0.05 & 0.1 & 0.25 & 0.5 & 0.75 & 0.9 & 0.95 & 0.99 \\
\hline \multirow{3}{*}{ Nonparametric } & CP & 0.8605 & 0.9561 & 0.9520 & 0.9461 & 0.9463 & 0.9564 & 0.9512 & 0.9232 & 0.2619 \\
& MSE & 0.00001 & 0.00005 & 0.0001 & 0.0003 & 0.0010 & 0.0035 & 0.0228 & 0.0158 & 0.0055 \\
& Length & 0.0100 & 0.0279 & 0.0456 & 0.0722 & 0.1258 & 0.2327 & 0.5922 & 0.4915 & 0.2149 \\
\hline \multirow{3}{*}{ Sectioning } & CP & 0.4825 & 0.8549 & 0.9065 & 0.9419 & 0.9489 & 0.9512 & 0.9291 & 0.8894 & 0.6206 \\
& MSE & 0.00012 & 0.00016 & 0.0002 & 0.0004 & 0.0010 & 0.0030 & 0.0097 & 0.0240 & 0.2354 \\
& Length & 0.0175 & 0.0308 & 0.0430 & 0.0720 & 0.1238 & 0.2136 & 0.3618 & 0.5103 & 0.9828 \\
\hline \multirow{3}{*}{ Jackknife } & CP & 0.7747 & 0.8422 & 0.8631 & 0.8898 & 0.8967 & 0.8918 & 0.8662 & 0.8380 & 0.7522 \\
& MSE & 0.00002 & 0.00007 & 0.0001 & 0.0004 & 0.0011 & 0.0033 & 0.0103 & 0.0227 & 0.1310 \\
& Length & 0.0156 & 0.0318 & 0.0447 & 0.0749 & 0.1299 & 0.2248 & 0.3971 & 0.5905 & 1.4186 \\
\hline
\end{tabular}

